\title{
IMPROVED CANTILEVERS FOR AFM THERMOMECHANICAL DATA STORAGE
}

\author{
Benjamin W. Chui, Timothy D. Stowe, Yongho S. Ju, Hyongsok T. Soh, Stephen C. Minne, \\ Kenneth E. Goodson, Calvin F. Quate, and Thomas W. Kenny \\ Departments of Electrical Engineering, Applied Physics, and Mechanical Engineering \\ Terman 551, Stanford University, California 94305-4021
}

\author{
H. Jonathon Mamin, Bruce D. Terris, Robert P. Ried, and Dan Rugar \\ IBM Research Division, Almaden Research Center, \\ 650 Harry Road, San Jose, California 95120-6099 \\ chui@leland.stanford.edu,mamin@almaden.ibm.com,kenny@cdr.stanford.edu
}

\section{INTRODUCTION}

In a previous report by Mamin et al [1], a new micromechanical approach to high-density data storage was described. In this technique, an atomic force microscope (AFM) tip is placed in contact with a rotating polycarbonate disk. A pulsed laser is used to heat the tip and thereby melt indentations into the disk, which has a glass transition temperature of $120-140{ }^{\circ} \mathrm{C}$. Another laser is used to sense the deflection of the cantilever as the tip passes over the recorded indentations. Bit densities up to $30 \mathrm{Gbit} / \mathrm{in}^{2}$ (50 times that of CD-ROM) have been demonstrated on a conventional AFM, and data rates up to $1.2 \mathrm{Mbit} / \mathrm{s}$ have been achieved on a rotating sample.

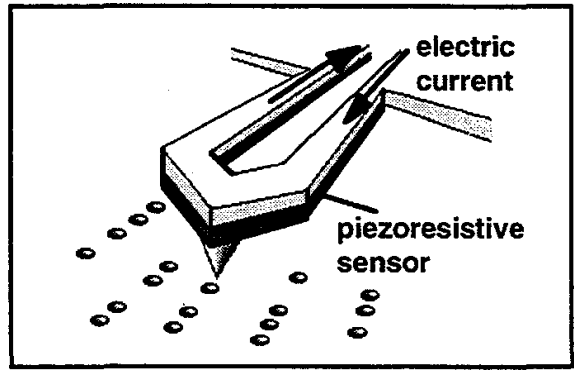

(a) Integrated piezoresistive sensor

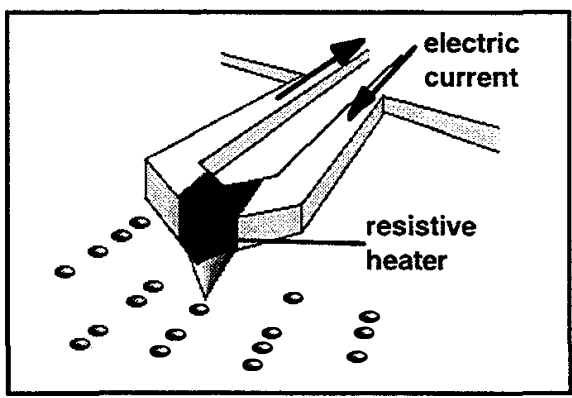

(b) Integrated resistive heater

Figure 1: Integration of read/write elements onto singlecrystal silicon cantilever for thermomechanical data storage.

One of the main problems with the existing read/write scheme is the need for external laser sources, which are bulky and require tedious alignment. As suggested in Figure 1, our ultimate goal is to develop an integrated read/write cantilever and eliminate the use of lasers. For this purpose we have developed single-crystal silicon cantilevers with piezoresistive elements for readback, based on the approach of Tortonese et al $[2,3]$. We have also made separate cantilevers with integrated resistive heaters for writing.

The ideal integrated cantilever will have to satisfy many conditions simultaneously. In order to read marks at densities of 20-50 Gbit/in ${ }^{2}$, it must have a tip that is sharp on the 500$1000 \AA$ scale. To achieve adequate signal-to-noise ratio, it needs a sensor capable of detecting a few angstroms of motion in the readback bandwidth. The cantilever must be soft-with a stiffness of $1 \mathrm{~N} / \mathrm{m}$ or less-to allow for operation at loads below $10^{-7} \mathrm{~N}$, which is necessary to avoid wear of the tip or sample. At the same time, the resonant frequency should be as high as possible, preferably in the $\mathrm{MHz}$ range. The combination of low stiffness and high frequency requires the cantilever to have low mass. For writing, an integrated element for heating the tip is required, and the thermal time constant should be as short as possible, preferably on the order of $1 \mu \mathrm{s}$.

In this work, we have not yet attempted to satisfy all these constraints simultaneously. Instead, we have focused on two areas. First, we have integrated piezoresistive sensors with cantilevers that are considerably softer than existing devices. With a cantilever of stiffness about $1 \mathrm{~N} / \mathrm{m}$, we have measured a deflection sensitivity of $1.6 \times 10^{-2} \AA / \sqrt{ } \mathrm{Hz}$ and a calculated resonant frequency of $250 \mathrm{kHz}$. To achieve this combination, we had to make considerably thinner cantilevers through some novel process development. Second, we have fabricated cantilevers with integrated electrical heaters, and have observed thermal time constants of roughly $40 \mu \mathrm{s}$. We have implemented both types of cantilevers on a rotating sample for demonstrating reading and writing without the use of lasers.

\section{CANTILEVER FABRICATION}

The fabrication process of the micro-cantilevers, shown in Figure 2, is adapted from reference [2]. The starting material is a silicon-on-insulator ( $\mathrm{SOI}$ ) wafer [4] with a 5-micron top silicon layer. In Figure $2 \mathrm{a}$, an $\mathrm{SF}_{6}$ plasma etch is used to undercut an oxide-resist mask to form a blunt tip, which is then sharpened by low-temperature oxidation (Figure $2 b$ ). In Figure $2 c$ the cantilever itself is patterned and a $1000 \AA$ oxide layer is grown over it. After this, boron is implanted through the oxide (Figure 2d) and subject to rapid thermal annealing. (Our process differs 
from reference [2] in that the implant comes after the oxidation step-the significance of this will be explained more fully in the next section.) In Figure $2 \mathrm{e}$, contact vias are etched through the oxide and aluminum is deposited and patterned to form electrical connections to the cantilever. Finally, a backside TMAH etch releases the cantilever (Figure 2f).

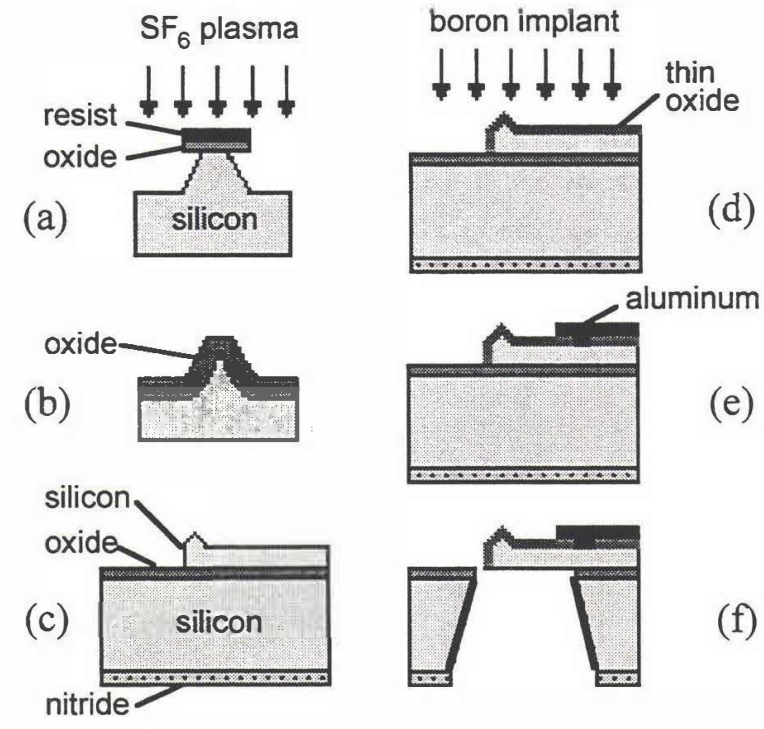

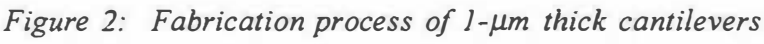

Figure 3 below shows an SEM micrograph of a fabricated cantilever and a close-up of the tip. The tip is seen to be very sharp, with a radius of curvature below $300 \AA$. Such a tip is quite suitable for reading and writing marks on a $1000 \AA$ scale.

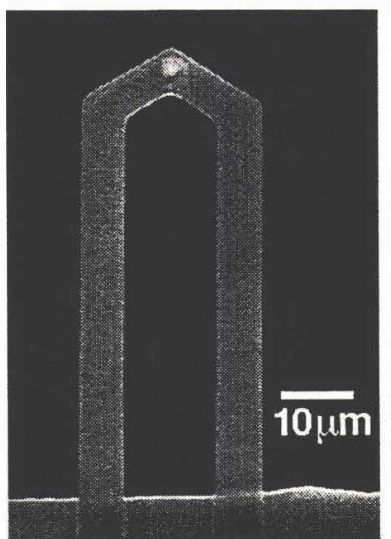

(a)

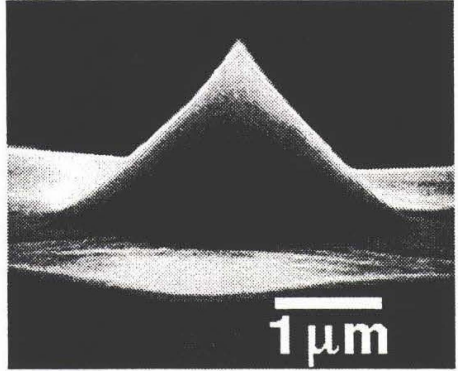

(b)
Figure 3: SEM images of (a) released cantilever and (b) tip

\section{INTEGRATED PIEZORESISTIVE SENSORS}

As explained above, it is necessary for the cantilever to be reasonably soft so that low forces can be maintained on the tip. To achieve low stiffness without sacrificing frequency, it is necessary to reduce the cantilever thickness considerably from the $2-4 \mu \mathrm{m}$ typically used in the process of Tortonese [2]. We have chosen to make $1-\mu \mathrm{m}$ thick cantilevers in this case. Implementing a piezoresistive sensor in such a thin cantilever is difficult, however. The reason is that the piezoresistor must be confined to a fraction of the cantilever thickness. Otherwise the compressive stress experienced by the piezoresistor on one surface cancels the tensile stress on the other, reducing the overall sensitivity.

We have developed a process for implantation and annealing of boron that prevents its diffusion throughout the cantilever thickness. The goal is to avoid post-implant hightemperature steps such as oxidation or annealing at $1000^{\circ} \mathrm{C}$, which are known to cause significant dopant diffusion. Our process therefore calls for the $1000 \AA$ oxide to be grown first at $1000{ }^{\circ} \mathrm{C}$, followed by a $40-\mathrm{keV}$ boron implant of $5 \times 10^{14} / \mathrm{cm}^{2}$ through the oxide. The implant is activated by a 10 -second rapid thermal anneal (RTA) step at $1000{ }^{\circ} \mathrm{C}$ and a 40 -minute furnace anneal at $800{ }^{\circ} \mathrm{C}$. A spreading resistance measurement of the implant profile [5] reveals a junction depth of roughly $0.4 \mu \mathrm{m}$ below the cantilever surface.

$\begin{array}{lll}\text { (a) "Control" cantilever (b) Heater-cantilever (c) Piezo-cantilever } & \end{array}$

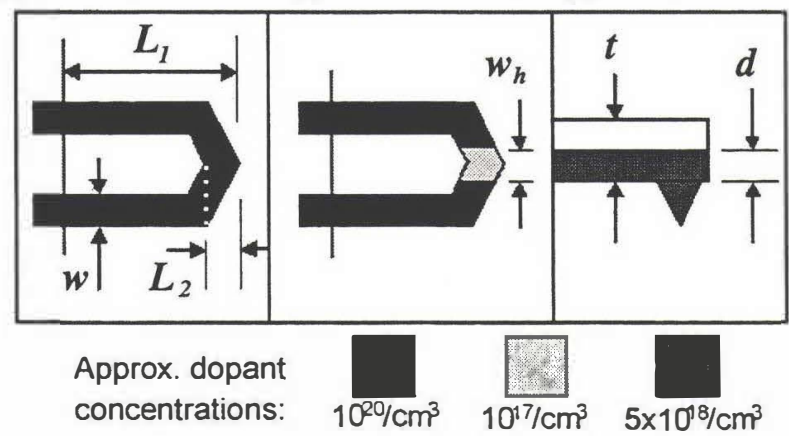

Figure 4: Diagram (not drawn to scale) showing cantilever dimensions, as well as differences between "control" cantilevers, heater-cantilevers, and piezoresistive cantilevers. Typical dimensions for our present design are $L_{I}=50-300 \mu \mathrm{m}$, $L_{2}=7 \mu \mathrm{m}, w=8 \mu \mathrm{m}, w_{h}=8 \mu \mathrm{m}$, and $t=1 \mu \mathrm{m}$.

A detailed calculation of the piezoresistive sensitivity of a cantilever can be found in reference [2]. To simplify the calculations, we have made the following assumptions:

(a) The width of the transverse part of the cantilever (where the tip is located) is small compared with the length of the cantilever (i.e. $L_{2}$ is much smaller than $L_{l}$ ). (Relevant cantilever dimensions are illustrated in Figure 4.)

(b) The piezoresistor doping is uniform through part of the cantilever thickness up to a step junction at depth $d$.

(c) The piezoresistor is uniformly distributed across the entire length of the cantilever. This reduces the sensitivity by a factor of 2 from the case where the resistor is concentrated at the base of the cantilever.

Under these conditions, the sensitivity is given by

$$
\frac{\Delta R}{R}=\frac{3 \pi_{L} E t \beta y}{4 L_{l}^{2}}
$$

where $\Delta R / R$ is the relative change in cantilever resistance,

$\pi_{L} \quad$ is the longitudinal coefficient of piezoresistivity of silicon,

$E$ is Young's modulus of silicon,

$t \quad$ is the thickness of the cantilever,

$L_{1}$ is the length of the cantilever, and

$y \quad$ is the displacement of the cantilever tip.

Here $\beta$ is an efficiency factor equal to 1 for the case where the piezoresistor is confined just to one surface of the 
cantilever. If assumption (b) is satisfied, the value of $\beta$ is given by $\beta=(1-d / t)$, where $d$ is the depth of the piezoresistive layer. A thicker piezoresistive layer implies a lower value of $\beta$.

Taking $\pi_{L}=5.7 \times 10^{-10} \mathrm{~m}^{2} / \mathrm{N}$ (in the [110] direction for boron-doped silicon at $\left.5 \times 10^{18} / \mathrm{cm}^{3}\right)[2], E=1.7 \times 10^{11} \mathrm{~N} / \mathrm{m}^{2}[2]$, $t=1 \mu \mathrm{m}$, and $d=0.4 \mu \mathrm{m}$ (implying $\beta=0.6$ ), we can calculate the sensitivity for different cantilever lengths. The theoretical sensitivity, which varies as the inverse of the square of the length, has been calculated with no fitting parameters and is shown as the dotted line in Figure 5.

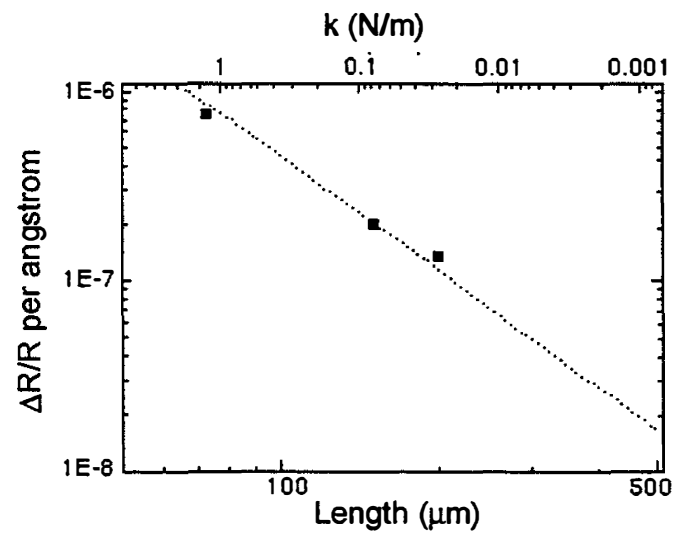

Figure 5: Sensitivity of piezoresistive cantilevers of various lengths. The dotted line represents the theoretical sensitivity calculated with no fitting parameters.

We have tested cantilevers of various lengths to determine their sensitivity. This was done by placing the cantilever on a piezoelectric actuator (which is part of a atomic force microscope) and modulating the cantilever support with the tip in contact with a fixed surface. Typical oscillation amplitudes were on the order of $1000 \AA$. The response was measured with a simple full bridge circuit based on a Burr-Brown INA103 instrumentation amplifier. This amplifier has an input noise of $1 \mathrm{nV} / \sqrt{\mathrm{Hz}}$ and a gain-bandwidth product of $100 \mathrm{MHz}$. We operated with a bridge supply voltage of $5 \mathrm{~V}$ (i.e. $2.5 \mathrm{~V}$ across each resistor) and a gain of 100 . Since the cantilever resistance was 5-30 k $\Omega$ (depending on length and doping concentration), the power dissipated in the cantilever was $1 \mathrm{~mW}$ or less.

The inferred values of $\Delta R / R$ per angstrom are plotted in Figure 5 . Test results show an excellent agreement with theory, with $\Delta R / R$ up to $0.75 \mathrm{ppm} / \AA$. In addition, these levels of sensitivity compare very favorably to those reported for other piezoresistive sensors, while our device has much lower stiffness. Table 1 shows a comparison between one of our cantilevers and one from ref. [2]. It is seen that while the two cantilevers have about the same $\Delta R / R$ per $\AA$, our cantilever exhibits a more favorable force figure of merit due to its lower spring constant.

\begin{tabular}{|l|c|c|c|c|c|c|}
\hline & $\begin{array}{c}L_{1} \\
(\mu \mathrm{m})\end{array}$ & $\begin{array}{c}t \\
(\mu \mathrm{m})\end{array}$ & $\begin{array}{c}\text { measured } \\
\Delta \mathrm{R} / \mathrm{R} \text { per } \AA\end{array}$ & $\begin{array}{c}k^{\mathrm{a}} \\
(\mathrm{N} / \mathrm{m})\end{array}$ & $\begin{array}{c}\mathrm{MDD}^{\mathrm{b}} \\
(\AA)\end{array}$ & $\begin{array}{c}\mathrm{FFOM}^{\mathrm{c}} \\
(\mathrm{nN})\end{array}$ \\
\hline This work & 75 & 1 & $7.5 \times 10^{-7}$ & 1.5 & 0.5 & 0.075 \\
\hline Ref.[2] & 170 & 3.8 & $8.5 \times 10^{-7}$ & 16.4 & 0.2 & 0.33 \\
\hline
\end{tabular}

Table 1: Comparison of piezoresistive cantilevers from this work and ref. [2]
Figure 6 shows the noise spectrum of a $75-\mu \mathrm{m}$ long cantilever. The observed noise floor of $1.6 \times 10^{-2} \AA / \sqrt{\mathrm{Hz}}$ is very close to the Johnson-limited noise floor of $1.3 \times 10^{-2} \AA / \sqrt{ } \mathrm{Hz}$. The $1 / f$ noise performance is quite good: with its knee at about $200 \mathrm{~Hz}$, the integrated $1 / f$ noise from $1 \mathrm{~Hz}$ to $200 \mathrm{~Hz}$ is only about $0.5 \AA$.

The inset of Figure 6 shows the response of the cantilever to a $100 \AA$ modulation applied with the AFM. The bandwidth used was $1 \mathrm{~Hz}-100 \mathrm{kHz}$, within which the minimum detectable displacement was less than $10 \AA$. Since data pits on the polycarbonate disk are typically $100-200 \AA$ in depth, piezoresistive readback of real data pits should be possible at this bandwidth.

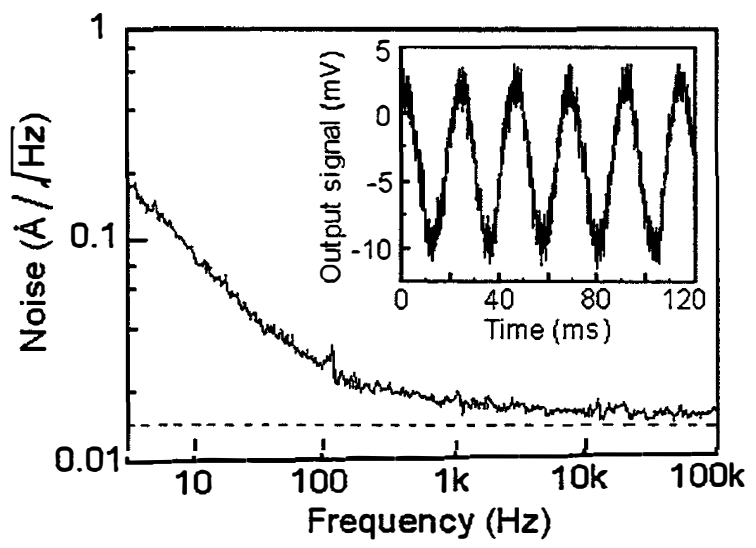

Figure 6: Noise spectrum of $75-\mu m$ piezoresistive cantilever. Theoretical Johnson noise floor is shown as dotted line. Figure 6 (Inset): Cantilever response to $100 \mathrm{~A}$ modulation.

Figure 7 shows a readback signal for one of our $75-\mu \mathrm{m}$ piezoresistive cantilevers operated on a spinning test sample with $600-\mathrm{nm}$ deep grooves. The bandwidth in this case was $300 \mathrm{kHz}$, and the linear velocity was approximately $120 \mathrm{~mm} / \mathrm{s}$. The waveform signal-to-noise ratio is excellent on these $600-\mathrm{nm}$ deep grooves, although of course the feature depth will be much smaller for real data pits. Still, this demonstrates the basic feasibility of integrated piezoresistive readback on a rotating sample.

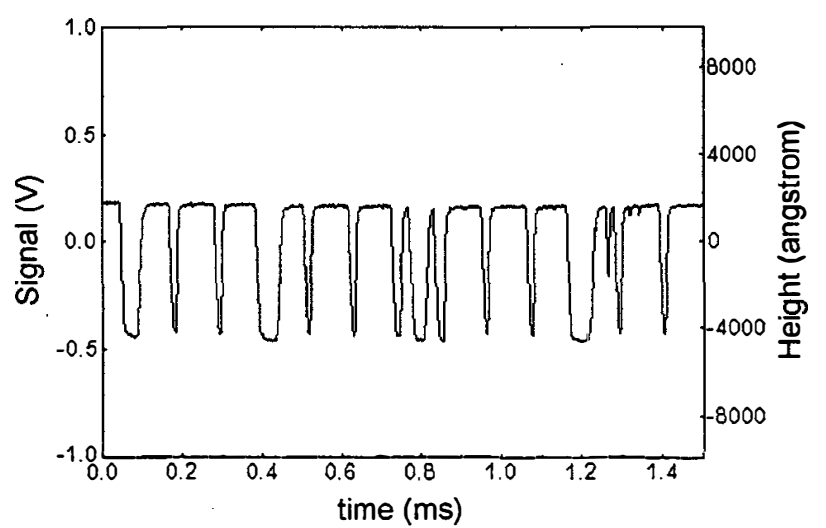

Figure 7: Readback signal obtained with 75- $\mu \mathrm{m}$ piezoresistive cantilever operated on spinning test sample with 600-nm deep grooves. 


\section{INTEGRATED HEATERS}

The present cantilevers are made of single-crystal silicon, which has the advantage that a wide range of resistivity is accessible through appropriate doping. This is very convenient for electrical heating, as it allows us to make the heating element and the leads of the same material. Another advantage is that the cantilevers are intrinsically low stress and have high thermal conductivity. Other approaches to micromachined heating elements include polysilicon [6] and nickel silicide [7].

We create the heaters by implanting the cantilever with boron except for a gap at the end which is several microns wide (denoted by $w_{h}$ in Figure 4 ). This lightly doped region forms the resistive heater. An electrical pulse applied to the cantilever will cause significant power dissipation in the heater region, giving rise to localized heating. After the pulse has ended, the heat is dissipated mainly by conduction down the legs to the support. The heavy implant concentration is about $10^{20} / \mathrm{cm}^{3}$ while the "heater" implant is about $10^{17} / \mathrm{cm}^{3}$. These boron implants are the same type as that used to form the piezoresistors, and can thus be carried out during the same process.

An analysis of the thermal properties of these cantilevers will be presented in the next section. We have demonstrated basic functionality of these cantilevers, as shown by the AFM micrograph in Figure 8. Sample data tracks were written with the resistive heater on a rotating polycarbonate sample. The writing was very reliable, and different-sized marks could be written by varying the pulse conditions. Typical pulse parameters were $16 \mathrm{~V}, 20 \mu \mathrm{s}$, with a $11 \mathrm{kHz}$ repetition rate.

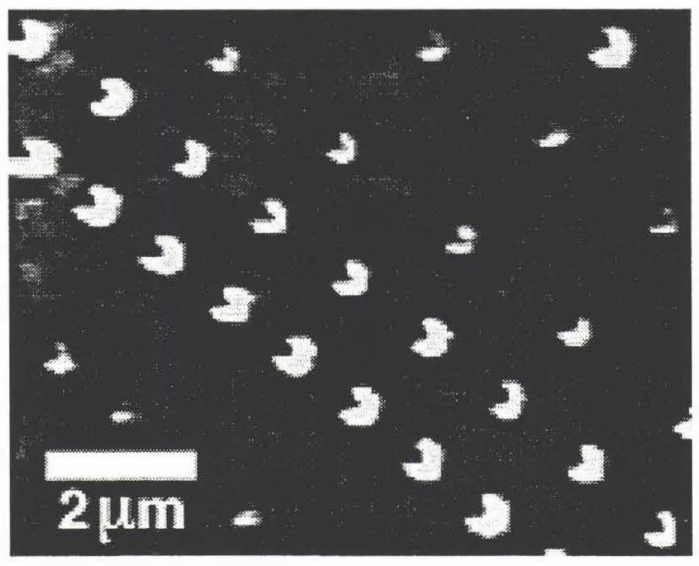

Figure 8: AFM image of sample data tracks

\section{THERMAL AND ELECTRICAL ANALYSIS}

We have measured the temperature variation of resistance of a $200-\mu \mathrm{m}$ long cantilever with an $8-\mu \mathrm{m}$ wide heater region. The cantilever chip was placed on a temperature-controlled chuck, and an HP4155A semiconductor parametric analyzer was used to apply a low test voltage across the cantilever and to measure the resulting current. The calculated resistance is shown in Figure 9, curve (a). Note that the observed resistance is the total resistance of the heater region and the cantilever legs. To find the resistance of the legs alone, we measured a "control" cantilever that has no heater region-what would have been the heater region is now doped as heavily as the legs and contributes very little to the overall resistance (see Fig. 4).
The resistance of such a "control" cantilever therefore corresponds to that of the legs, and its measured value at different temperatures is shown in curve (b) of Figure 9. Subtracting (b) from (a) gives curve (c), which represents the resistance of the heater region alone. Curve (c) will be used in conjunction with another measurement described below (see Figure 10) to help estimate the transient temperature behavior of the heater.

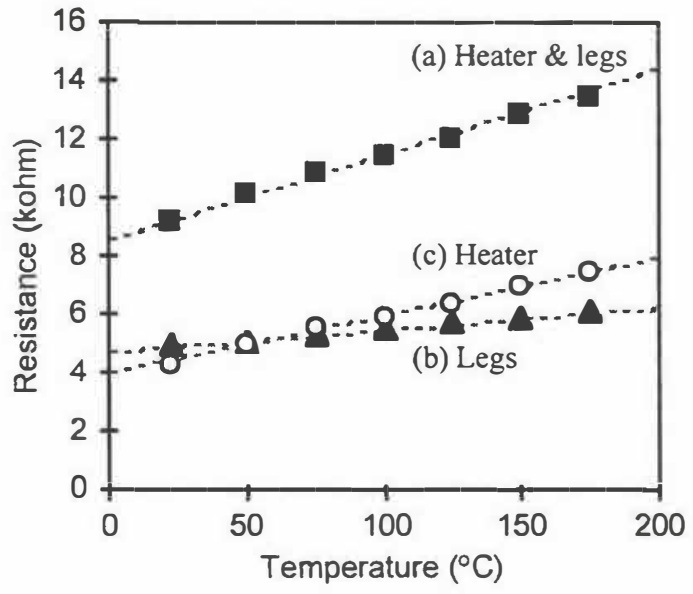

Figure 9: Resistance vs. temperature of 200- $\mu \mathrm{m}$ cantilever with 8- $\mu \mathrm{m}$ heater. Curve (a) shows total resistance of cantilever; curve (b) shows resistance of cantilever legs; curve (c) shows derived resistance of heater region alone (i.e. difference between (a) and (b)).

Figure 9 implies positive temperature coefficients of resistance for the legs and the heater on the order of $0.1 \% /{ }^{\circ} \mathrm{C}$ and $0.3 \% /{ }^{\circ} \mathrm{C}$ respectively. One would therefore expect that when a voltage pulse is applied to the cantilever, the resulting current would decrease as the cantilever heats up and increases in resistance. After the pulse, the resistance would gradually decrease to its room-temperature value as the cantilever cools. If a small DC test voltage is present across the cantilever after the pulse has ended, the resulting current would be expected to rise and approach a final room-temperature value as the cantilever cools. By measuring the instantaneous current during and after a voltage pulse, we can infer the transient temperature behavior of a heater-cantilever. The cooling rate of the heater corresponds to the thermal time constant and determines how fast successive marks can be written, i.e. the data rate.

In one experiment, a $11 \mathrm{~V}, 5 \mu$ s heating pulse was applied across a cantilever similar to the one used to obtain Figure 9. The pulse was superimposed on a $1 \mathrm{~V} \mathrm{DC}$ offset. The value of $1 \mathrm{~V}$ was chosen so as not to cause noticeable heating. The applied pulse is shown in Figure 10a. The instantaneous current that flowed through the cantilever was determined from the voltage across a $100 \Omega$ series resistor, and the oscilloscope trace is reproduced in Figure $10 \mathrm{~b}$. As predicted, the current decayed during the pulse, indicating a rise in heater temperature. The time variation of the heater resistance can be derived, and the heater temperature can be estimated using the data in Figure $10 \mathrm{~b}$ as well as the resistivity-versus-temperature data in Figure 9, curve (c). The resulting estimate is shown in Figure 10c. This type of electrical measurement has previously been used for characterizing micromachined elements [6] as well as commercial AFM cantilevers [8] 


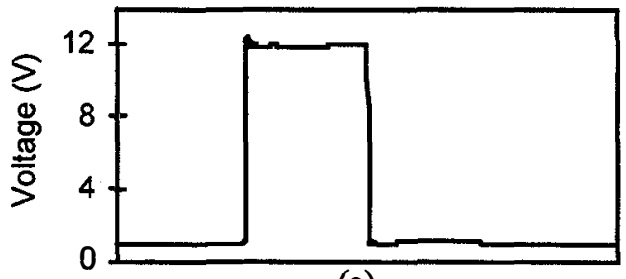

(a)
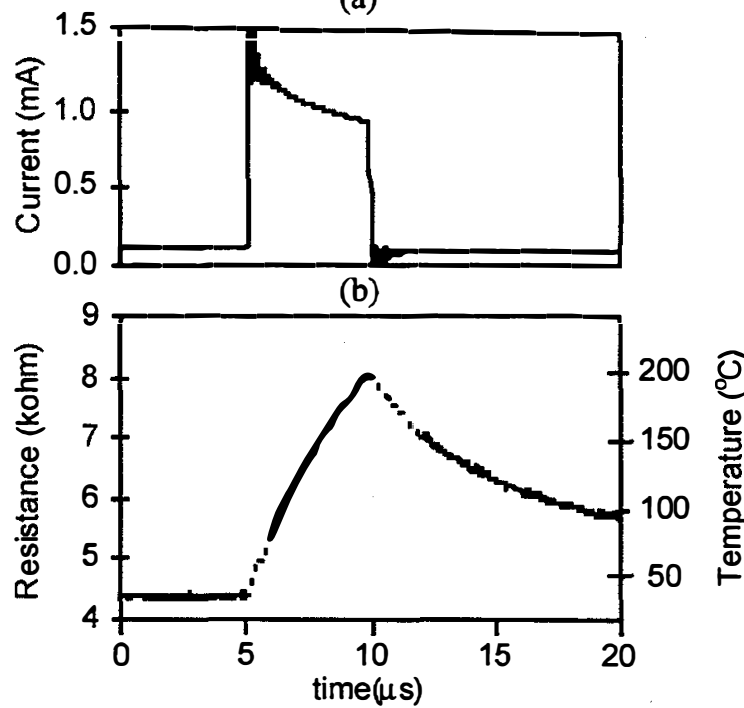

(c)

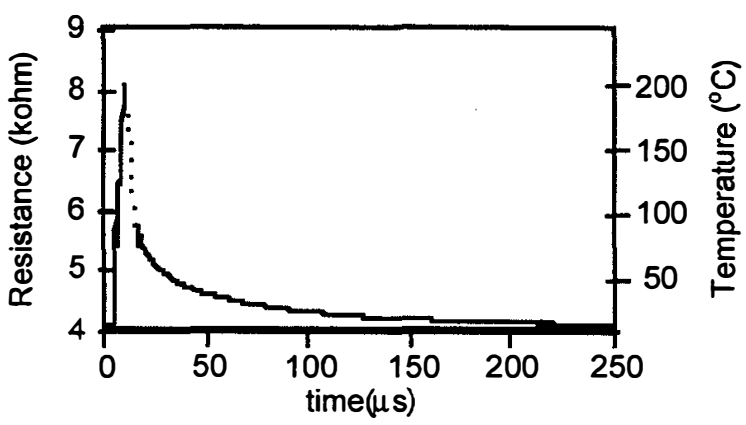

(d)

Figure 10: Electrical measurements for estimating heater temperature during heating pulse. Curve (a): Applied voltage pulse (amplitude is $12 \mathrm{~V}$ at time $5-10 \mu \mathrm{s}$ and $1 \mathrm{~V}$ at all other times). Curve (b): Measured current determined from voltage across 100- $\Omega$ series resistor. Curve(c): Derived heater resistance and temperature, on 20- $\mu$ s time scale. The sections of the curve at 5-6 $\mu \mathrm{s}$ and 10-12 $\mu$ s represent interpolated data, since actual data was obscured by excessive ringing. Curve(d): Derived heater resistance and temperature on longer time scale of $250 \mu \mathrm{s}$, showing cooling time constant. Dotted lines show interpolated data for curves (c) and (d).

To investigate the cooling time constant, we measured the current that flowed after the pulse as a result of the $1 \mathrm{~V}$ offset voltage. The derived heater resistance and temperature is indicated in Figure 10, curve (d). As expected, the resistance started out from a relatively high value just after the pulse has ended and, as the heater cooled, gradually decreased towards its room-temperature value. It is seen that the thermal time constant is about $40 \mu \mathrm{s}$, compared with $350-450 \mu \mathrm{s}$ for a commercial silicon cantilever without a localized heater [8].
Unfortunately, the circuitry for measuring the current produces excessive ringing for several microseconds after the pulse, making it difficult to extract meaningful data during that period. This calls for a supplementary method of measurement that provides adequate time resolution under such conditons. One possible choice is laser thermometry.

Laser thermomery makes use of the fact that the optical reflectance of most materials varies with temperature. While this type of measurement is most commonly used with metals with up to picosecond-level resolution, in some cases it has been adapted for investigating semiconductor devices [9] such as SOI power transistors [10]. In our experimental setup, a lowpower laser beam is focused on a heater-cantilever, and the reflected power is measured at different points in time. The results are used to estimate the heater temperature and the thermal time constant.

Because of the small laser spot size (on the order of $3 \mu \mathrm{m}$ ), laser thermometry will allow us to investigate the spatial distribution of temperature along the cantilever with sufficient time resolution to follow the propagation of the heat pulse. In future experiments, we will use this technique to confirm thermal models of heat propagation in these cantilevers.

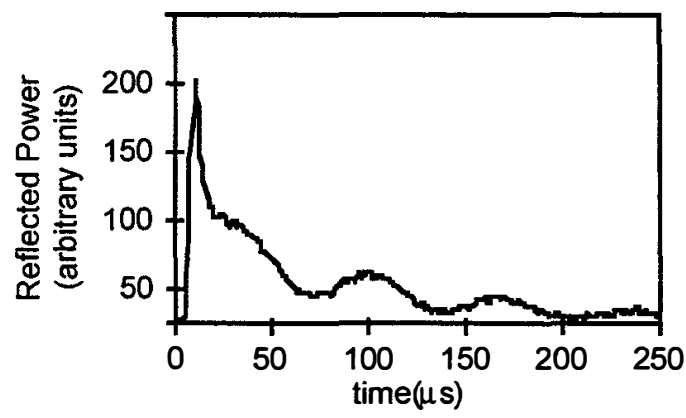

Figure 11: Reflected laser power from heater-cantilever, under same conditions as Figure 10.

Figure 11 shows the reflected laser power from a location near the end of the cantilever in response to a heating pulse. This curve shows two distinct phenomena: an oscillation at 14 $\mathrm{kHz}$, and a decay with a time constant of about $40 \mu \mathrm{s}$. We believe that the overall decay is related to the temperature coefficient of the reflectivity of the cantilever; the time constant for this decay is consistent with the resistance measurements. In addition, we believe that the oscillation is due to a thermally induced vibration; the frequency of this oscillation is consistent with the resonant frequency of the cantilever. The cantilever has a $1000-\AA$ oxide layer on top of a $1-\mu \mathrm{m}$ silicon layer, so heating ought to produce a differential thermal expansion.

To confirm this hypothesis, we have used a vibrometer [11] to study the pure mechanical behavior of a cantilever in response to a heating pulse. In this technique, a laser interferometer is used to measure the motion of the cantilever tip with angstrom-level resolution. The output of the vibrometer operated in velocity mode is shown in Figure 12. A decaying sinusoidal oscillation is observed, confirming the mechanical origins of the oscillation in Figure 11. From Figure 12 the initial amplitude of the vibration can be estimated at roughly $350 \AA$, with a frequency of about $15 \mathrm{kHz}$. The difference in observed frequency between Figures 11 and 12 is most likely due to a slight mismatch in the length and thickness of the two cantilevers being measured. 


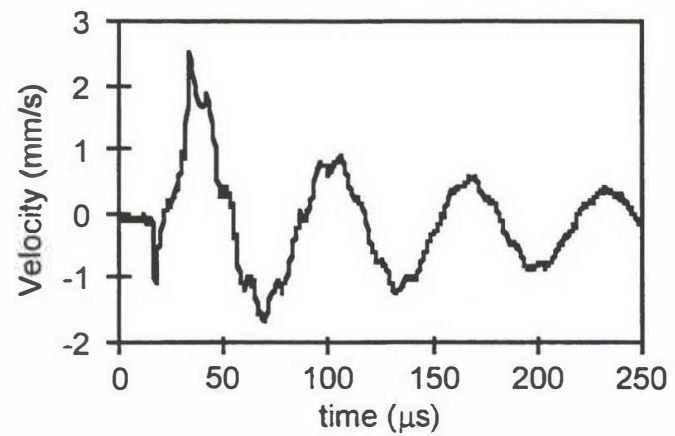

Figure 12: Vibrometer output signal showing mechanical vibration of cantilever tip.

As supplementary techniques to electrical measurements, laser thermometry and vibrometry make it possible to characterize the behavior of cantilevers in detail. Laser thermometry can provide spatially and temporally resolved measurements of cantilever temperature; laser vibrometry can do the same with regard to cantilever motion. Together, these two types of measurement will be useful for the development of improved cantilevers for thermomechanical data storage.

\section{CONCLUSIONS}

In this report, we have described two novel capabilities of AFM cantilevers. First, we have worked to add integrated sensing functionality onto low-stiffness cantilevers with integrated tips. A new process was developed which allows for considerably thinner cantilevers than previously possible, and should be scaleable to even thinner cantilevers. The measured sensitivity is in excellent agreement with predictions, so that we can calculate with confidence the type of cantilever needed to achieve a given level of performance. The current cantilevers are adequate to detect $100-200 \AA$ deep features in a roughly 100 $\mathrm{kHz}$ bandwidth. They also show excellent $1 / f$ noise behavior.

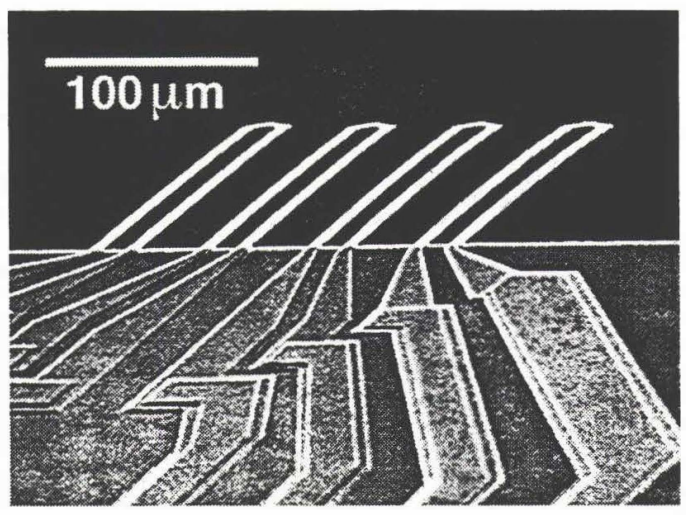

Figure 13: SEM image of fabricated cantilever array

A heater process was also developed on separate cantilevers which allows for thermal writing without the need for a laser. Electrical and laser thermometry measurements were made which indicate for our $200-\mu \mathrm{m}$ heater-cantilevers a thermal time constant of about $40 \mu \mathrm{s}$, or 10 times shorter than for commercial silicon cantilevers. One of the advantages of micromachining is that the components are batch fabricated, so that arrays of tips can be easily made. Figure 13 shows an array of four cantilevers fabricated on the same wafer as the other cantilevers. The operation of cantilevers in parallel has already been demonstrated in AFM microscopy [12]. Ultimately, the same concept may be used in AFM data storage to improve the level of performance.

\section{ACKNOWLEDGMENTS}

We would like to thank J.D. Plummer of Stanford and L.S. Fan of IBM for providing valuable insight into the electrical characteristics of the heater-cantilever. We are also grateful to M. Tortonese of Park Scientific Instruments for helpful discussions, and to O.W. Käding for his work on the laser thermometry setup. We would like to acknowledge the support of an IBM Cooperative Fellowship, the National Science Foundation CAREER Program (ECS-9502046), the National Science Foundation Instrumentation for Materials Research Program (DMR-9504099), and the Charles Lee Powell Foundation. The work of H.J. Mamin and R.P. Ried is supported in part by ARPA contract number DABT63-95-C-0019. We would finally like to thank the National Science Foundation for its generous support of travel expenses for this conference.

\section{REFERENCES}

1. H.J. Mamin, L.S. Fan, S. Hoen, D. Rugar, "Tip-based data storage using micromechanical cantilevers," Sensors and Actuators $A, 48$ (1994), pp. 215-219.

H.J.Mamin, D.Rugar, "Thermomechanical writing with an atomic force microscope tip," Appl. Phys. Lett., 61(1992), p.1003-5.

2. M. Tortonese, Ph.D thesis, 1994 (unpublished)

3. M. Tortonese, R.C. Barrett, and C.F. Quate, "Atomic resolution with an atomic force microscope using piezoresistive detection," Appl. Phys. Lett., 62, 8 (1993), pp. 834-6.

4. SOI wafers purchased from IBIS Corporation, phone (408) 662-9100.

5. Spreading resistance profile measurement by Solecon Laboratories, 2241 Paragon Drive, San Jose, California 95131.

6. C.H. Mastrangelo, J.H.-J. Yeh, R.S. Muller, "Electrical and optical characteristics of vacuum-sealed polysilicon microlamps." IEEE Trans. on Elec. Dev., 39, 6 (1992), pp. 1363-75.

7. J.H. Das, N.C. MacDonald, "Micromachined field emission cathode with an integrated heater," J. Vac. Sci. Technol. B, 13, 6 (1995), pp. 2432-5.

8. H.J. Mamin, "Thermal writing using a heated atomic force microscope tip," submitted to Appl. Phys. Lett. (1996).

9. P.W. Epperlein, "Micro-temperature measurements on semiconductor laser mirrors by reflectance modulation: A newly developed technique for laser characterization," Jpn. J. Appl. Phys., 32, 12A (1993), pp. 5514-22.

10. K.E. Goodson, Y.S. Ju, O.W. Käding, "Short-timescale thermal mapping of semiconductor devices," submitted to IEEE Elec. Dev. Lett. (1996).

11. Polytec Laser Vibrometer OVF-501-0, Polytec P.I. Inc., 3152 Redhill Suite 110, Costa Mesa, California 92626.

12. S.C. Minne, S.R. Manalis, C.F. Quate, "Parallel atomic force microscopy using cantilevers with integrated piezoresistive sensors and integrated piezoelectric actuators," Appl. Phys. Lett. 67, 26, (1993), pp. 3918-20. 